\title{
A Majority-Based Aggregation Operator to Represent Emotional States
}

\author{
A.G. Lopez-Herrera ${ }^{1}$ M.A. Muñoz ${ }^{2}$ M.I. Viedma-del-Jesus ${ }^{2}$ E. Herrera-Viedma ${ }^{3}$ \\ ${ }^{1}$ Dept. Computer Sciences, University of Jaen, 23071-Jaen, Spain. aglopez@ujaen.es \\ ${ }^{2}$ Dept. Personality, University of Granada, 18071-Granada, Spain. \{mamuoz, iviedma\}@ugr.es \\ ${ }^{3}$ Dept. Computer Sciences \& A.I., University of Granada, 18071-Granada, Spain. viedma@decsai.ugr.es
}

\begin{abstract}
The Self-Assessment Manikin (SAM) is an universally used instrument of pictographic non verbal measure which is used in many emotional studies in the field of the Psychology. In these emotional studies the usual aggregation operator is the classical mean. In this paper, a new aggregation operator is proposed. This new operator, which is based on the majority concept tries to solve the distribution problems of the mean operator. This new aggregation operator is applied in a real emotional study, in which the SAM is used, achieving better results than the classical mean operator.
\end{abstract}

Keywords: SAM, Aggregation Operator, Majority Concept.

\section{Introduction}

The Self-Assessment Manikin (SAM) is an universally used instrument of pictographic non verbal measure which requires no language and therefore is easy to administer in different cultures [12][13]. The SAM has been use in different studies about emotion, this has directed the construction of different emotional instruments. This method provides information in three general emotional dimensions: valence, arousal and dominance associated with a person's affective reaction to a wide variety of stimuli (pictures, movies, natural-sounds...). SAM consists of human-like figures that embody the dimensions of valence, arousal, and dominance, with five figures representing five intensity levels within each dimension. SAM's valence rating ranges from a figure with a pronounced frown to figure with a large smile (Figure 1), arousal rating ranges from a figure that looks drowsy to a figure that appears agitated (Figure 2), and the submissivenessdominance (not in contro-in control) rating ranges from a very small figure to a very large figure (Figure 3). SAM visually represents the three dimensions and was designed as an alternative to the sometimes-cumbersome verbal self-report measures [11]. This subjective assessment method has been extensively validated and is widely used in cue reactivity research [2][3], on different populations like dug addict [1], psychopath [15] and common population [17]. In these studies [1]-[3][15][17], stimuli are slides from IAPS (International Affective Picture System) [5]. These slides are rated on the SAM scales and mean ratings for each dimension were computed for each slide. These mean ratings are used to compare the different populations.

The mean operator is a common aggregation operator that produces reasonable results, but, at the same time, when the items to aggregate have cardinality $>1$ they may produce distribution problems. For example, let us consider the aggregation of the following values $\{0.7,0.7,0.7,0.7,0.5,0.4,0.1,0.1,0.1\}$. If we analyze this example, $55 \%$ (the majority) of values are over than 0.5 and $44 \%$ are 0.7 . Because of this, the result should be higher than 0.5 and lower than 0.7 , however the result of the mean operator is 0.44 . These distribution problems might produce erroneous comparisons among populations.

There exists other aggregation operators that use the rates given by the majority to produce a overall rate. We think that using these majoritybased aggregation operators better results would be achieved. The different populations would be compared using the opinion or rate given by the majority of the members of the population, rather than the mean of the rates.

In this paper, we propose a new majority-based aggregation operator and its application to SAM to get better results. To do that, this paper is structured as follows: in Section 2, the ordinal and the 2tuple fuzzy linguistic approaches are presented and the group decision making process is introduced; in Section 3 the new majority-based aggregation operator is proposed and tested with participants from a real psychological study; and finally, some conclusions are drawn in Section 4. 


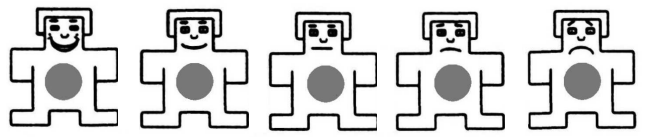

Fig. 1: Valence dimension.

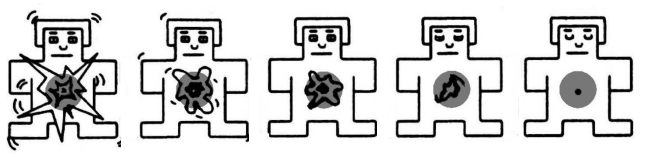

Fig. 2: Arousal dimension.

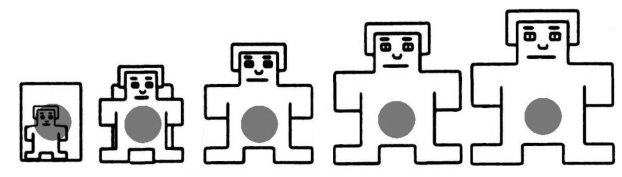

Fig. 3: Dominance dimension.
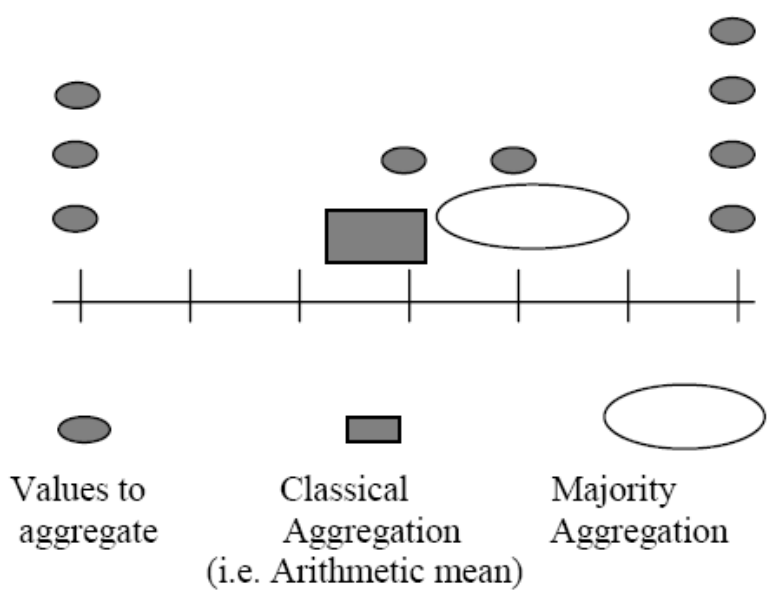

Fig. 4: Graphics representation using the classical aggregation and majority aggregation.

\section{Preliminaries}

In this section, the ordinal and the 2-tuple fuzzy linguistic approaches are introduced, and the group decision making process is described.

\subsection{The Ordinal Fuzzy Lin- guistic Approach}

The ordinal fuzzy linguistic approach is a fuzzy approximate technique appropriate to deal with qual- itative aspects of problems [9]. It models linguistic information by means of ordinal linguistic labels supported by a linguistic variable [18]. A linguistic variable is defined by means of a syntactic rule and a semantic rule. In an ordinal fuzzy linguistic approach the syntactic rule is defined by considering a finite and totally ordered label set $\mathcal{S}=\left\{s_{i}\right\}, i \in\{0, \ldots, \mathcal{G}\}$ in the usual sense, i.e., $s_{i} \geq s_{j}$ if $i \geq j$, and with odd cardinality (such as 7 or 9 labels), where the mid term represents an assessment of "approximately 0.5 ", and the rest of the terms being placed symmetrically around it. The semantics of the linguistic term set is established from the ordered structure of the term set by considering that each linguistic term for the pair $\left(s_{i}, s_{\mathcal{G}-i}\right)$ is equally informative. In any linguistic approach we need operators of management of linguistic information, such as:

- Minimization operator,

$$
\operatorname{MIN}\left(s_{a}, s_{b}\right)=s_{a} \text { if } a<=b
$$

- Maximization operator

$$
M A X\left(s_{a}, s_{b}\right)=s_{a} \text { if } a>=b
$$

- Negation operator

$$
N E G\left(s_{i}\right)=s_{j} \mid j=\mathcal{G}-i
$$

- Aggregation operators, for example the LOWA operator [7].

\subsection{The 2-Tuple Fuzzy Lin- guistic Approach}

Usually, to define a fuzzy linguistic model we must establish its representation model of linguistic information and its computational model to combine linguistic information. In such a way, in [8] was introduced the 2-tuple fuzzy linguistic model by defining both its representation model and its computational model.

Definition 1. Let $\mathcal{S}=\left\{s_{0}, \ldots, s_{\mathcal{G}}\right\}$ be a linguistic term set and $\beta \in[0, \mathcal{G}]$ a value supporting the result of a symbolic aggregation operation, then the 2-tuple that expresses the equivalent information to $\beta$ is obtained with the following function:

$$
\begin{aligned}
& \Delta:[0, \mathcal{G}] \longrightarrow \mathcal{S} \times[-.5, .5) \\
& \Delta(\beta)=\left(s_{i}, \alpha\right),\left\{\begin{array}{cc}
s_{i} & i=\operatorname{round}(\beta) \\
\alpha=\beta-i & \alpha \in[-.5, .5)
\end{array}\right.
\end{aligned}
$$

where round $(\cdot)$ is the usual round operation, $s_{i} \in \mathcal{S}$ has the closest index label to " $\beta$ " and " $\alpha$ " is the value of the symbolic translation. 
Proposition 1. Let $\mathcal{S}=\left\{s_{0}, \ldots, s_{\mathcal{G}}\right\}$ be a linguistic term set. There is always a $\Delta^{-1}$ function, such that, from a 2-tuple $\left(s_{i}, \alpha\right)$ it returns its equivalent numerical value $\beta \in[0, \mathcal{G}] \subset \mathcal{R}$.

$$
\begin{gathered}
\Delta^{-1}: \mathcal{S} \times[-.5, .5) \longrightarrow[0, \mathcal{G}] \\
\Delta^{-1}\left(s_{i}, \alpha\right)=i+\alpha=\beta
\end{gathered}
$$

Remark 1. We should point out that the conversion of a linguistic term into a linguistic 2-tuple consists of adding a value 0 as value of symbolic translation: $s_{i} \in \mathcal{S} \Longrightarrow\left(s_{i}, 0\right)$.

On the other hand, the 2-tuple fuzzy linguistic computational model carries out processes of computing with words in a precise way when the linguistic term sets are symmetrically and uniformly distributed. This computational model presents different techniques to manage the linguistic information $[8]$ :

- Comparison of 2-tuples: The comparison of linguistic information represented by 2 -tuples is carried out according to an ordinary lexicographic order. Let $\left(s_{k}, \alpha_{1}\right)$ and $\left(s_{l}, \alpha_{2}\right)$ be two 2-tuple:

- if $k<l$ then $\left(s_{k}, \alpha_{1}\right)$ is smaller than $\left(s_{l}, \alpha_{2}\right)$

- if $k=l$ then

$*$ if $\alpha_{1}=\alpha_{2}$ then $\left(s_{k}, \alpha_{1}\right),\left(s_{l}, \alpha_{2}\right)$ represent the same information

$*$ if $\alpha_{1}<\alpha_{2}$ then $\left(s_{k}, \alpha_{1}\right)$ is smaller than $\left(s_{l}, \alpha_{2}\right)$

* if $\alpha_{1}>\alpha_{2}$ then $\left(s_{k}, \alpha_{1}\right)$ is bigger than $\left(s_{l}, \alpha_{2}\right)$

- Negation of 2-tuple is defined as:

$$
N e g\left(s_{i}, \alpha\right)=\Delta\left(\mathcal{G}-\Delta^{-1}\left(s_{i}, \alpha\right)\right) .
$$

- Aggregation of 2-tuples: Using the function $\Delta$ and $\Delta^{-1}$ any aggregation operator can be easily extended for dealing with linguistic 2 tuples. Some examples are presented in [8].

\subsection{The Group Decision Mak- ing Process}

Group decision-making (GDM) problems can be defined as decision situations where 1) there are two or more experts who are characterized by their own ideas, attitudes, motivations, and knowledge, 2) there is a problem to be solved, and 3) they try to achieve a common solution [4]. To obtain a common value for all experts, there exist diverse

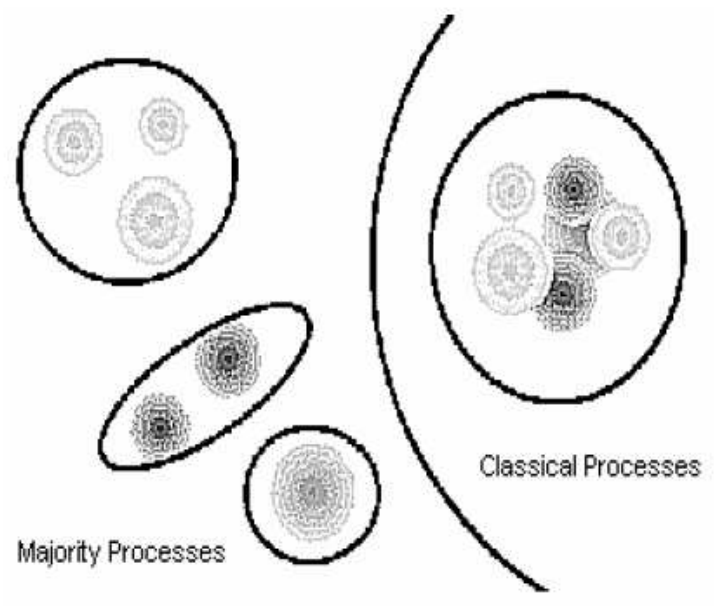

Fig. 5: Representation of the Majority and Classical Processes.

fuzzy approaches in which are realized an aggregation of the individual opinions guided generally by the concept of majority, where majority is defined as a collective evaluation in which the opinions of most of the experts involved in the decision problem are considered. In these approaches the result is not necessarily unanimous, but a solution must be obtained with agreement among a fuzzy majority of the decision makers [6][14].

Majority processes arise because the most common aggregation operators over-emphasize the opinion of the minority as the expense of those of the majority creating an aggregation that may be considered imprecise for the majority. These are called distribution problems. For example, let us consider the aggregation of the following labels: highest, highest, highest, highest, high, medium, lowest, lowest, lowest, of the set $\mathcal{S}=\{$ lowest, very_low, low, medium, high, very_high, highest\}, where $s_{0}=$ lowest, $s_{1}=$ very_low, $s_{2}=$ low, $s_{3}=$ medium, $s_{4}=$ high, $s_{5}=$ very_high, $s_{6}=$ highest. If we analyze this example (Figure 4), $55 \%$ of labels are higher than medium and $44 \%$ are the highest label. Due to this distribution, the result should be higher than medium and lower than highest, because we obtain a value that represents the majority and at the same time the minority. However, classic aggregation processes obtain inferior values that are close to medium as in Figure 4, producing distribution problems.

These distribution problems result from the process through which the items are considered. Classical aggregation considers items in an independent manner while majority processes consider 
groups of items with different cardinalities. Figure 5 graphically represents this difference. This example has 6 elements to aggregate. Classic aggregation processes all these elements independently while the majority aggregation first groups items by their similarities and then aggregates. In the above example, classical operators manage nine sets with cardinality 1 , nevertheless, the majority process considers four sets with cardinality 4, 1, 1 and 3 respectively.

\section{The 2-Tuple Majority-Based Aggregation Operator MA- LOWA $_{2 t}$}

In this section we propose a new majority-based aggregation operator, called MA-LOWA $2 t$, which is a 2-tuple extension of the MA-OWA operator [16] using the LOWA operator [7].

First, we have to define the 2-tuple-based LOWA operator [10].

Definition 2. Let $\left\{\left(a_{1}, \alpha_{1}\right), \ldots,\left(a_{m}, \alpha_{m}\right)\right\}$ be a set of 2-tuple assessments to aggregate, then the $\mathrm{LOWA}_{2 t}$ operator $\phi_{2 t}$ is defined as:

$$
\begin{gathered}
\phi_{2 t}\left(\left(a_{1}, \alpha_{1}\right), \ldots,\left(a_{m}, \alpha_{m}\right)\right)=W \cdot B^{T}= \\
C_{2 t}^{m}\left\{w_{k}, b_{k}, k=1, \ldots, m\right\}= \\
=w_{1} \otimes b_{1} \oplus\left(1-w_{1}\right) \otimes C_{2 t}^{m-1}\left\{\beta_{h}, b_{h}, h=2, \ldots, m\right\}
\end{gathered}
$$

where $b_{i}=\left(a_{i}, \alpha_{i}\right) \in(\mathcal{S} \times[-.5, .5)), W=$ $\left[w_{1}, \ldots, w_{m}\right]$ is a weighting vector, such that $t_{i} \in$ $[0,1]$ and $\sum_{i} w_{i}=1, \beta_{h}=\frac{w_{h}}{\sum_{2}^{m} w_{k}}, h=2, \ldots, m$, and $B$ is the associated ordered 2-tuple vector. Each element $b_{i} \in B$ is the i-th largest 2-tuple in the collection $\left\{\left(a_{1}, \alpha_{1}\right), \ldots,\left(a_{m}, \alpha_{m}\right)\right\}$, and $C_{2 t}^{m}$ is the convex combination operator of $m$ 2-tuples. If $w_{j}=1$ and $w_{i}=0$ with $i \neq j \forall i, j$, the convex combination is defined as: $C_{2 t}^{m}\left\{w_{i}, b_{i}, i=1, \ldots, m\right\}=$ $b_{j}$. And if $m=2$ then it is defined as:

$$
\begin{gathered}
C_{2 t}^{2}\left\{w_{l}, b_{l}, l=1,2\right\}= \\
=w_{1} \otimes b_{j} \oplus\left(1-w_{1}\right) \otimes b_{i}=\Delta(\lambda),
\end{gathered}
$$

where $\lambda=\Delta^{-1}\left(b_{i}\right)+w_{1} \cdot\left(\Delta^{-1}\left(b_{j}\right)-\Delta^{-1}\left(b_{i}\right)\right)$, $b_{j}, b_{i} \in \mathcal{S} \times[-.5, .5),\left(b_{j} \geq b_{i}\right), \lambda \in[0, \mathcal{G}]$.

MA-LOWA $2 t$, like MA-OWA is a neat OWA and their weights are calculated in functions of the cardinality of the elements to aggregate and they are independent of the order. The operator is defined as follows:

Definition 3. Let $\left\{\left(a_{1}, \alpha_{1}\right), \ldots,\left(a_{m}, \alpha_{m}\right)\right\}$ be a set of 2-tuple assessments to aggregate using a majority-based scheme, then the MA-LOWA $2 t$ operator $\phi_{2 t}^{M A}$ is defined as:

$$
\begin{gathered}
\phi_{2 t}^{M A}\left(\left(a_{1}, \alpha_{1}\right), \ldots,\left(a_{m}, \alpha_{m}\right)\right)=W \cdot B^{T} \\
=C_{2 t}^{n}\left\{w_{k}, b_{k}, k=1, \ldots, n\right\}=
\end{gathered}
$$

$=w_{1} \otimes b_{1} \oplus\left(1-w_{1}\right) \otimes C_{2 t}^{n-1}\left\{\beta_{h}, b_{h}, h=2, \ldots, n\right\}$

where $m$ and $n$ are the number of items and number of groups to be aggregated respectively, $w_{i} \in[0,1]$ with $\sum_{i=1}^{n} w_{i}=1$ and $b_{i}$ is the i-th element of $\left\{\left(a_{1}, \alpha_{1}\right), \ldots,\left(a_{m}, \alpha_{m}\right)\right\}$ that is ordered in ascender order by cardinalities. On the other hand, $w_{i}=$ $f_{i}\left(b_{1}, \ldots, b_{n}\right)=\frac{\gamma_{i}^{\delta_{\min }}}{\theta_{\delta_{\max }} \cdot \theta_{\delta_{\max -1} \cdot \ldots \cdot \theta_{\delta_{\min +1}} \cdot \theta_{\delta_{\min }}}}+$ $\frac{\gamma_{i}^{\delta_{\min +1}}}{\theta_{\delta_{\max }} \cdot \theta_{\delta_{\max -1}} \cdot \cdots \cdot \theta_{\delta_{\min +1}}}+\ldots+\frac{\gamma_{i}^{\delta_{\max }}}{\theta_{\delta_{\max }}}$

with

$$
\gamma_{i}^{k}=\left\{\begin{array}{l}
1 \quad \text { if } \delta_{i} \geq k \\
0 \quad \text { otherwise }
\end{array}\right.
$$

and

$$
\theta_{i}=\left\{\begin{array}{lr}
z+1 & \text { if } i \neq \delta_{\min } \\
z & \text { otherwise }
\end{array}\right.
$$

where $z=$ number of items with cardinality $\geq i$.

$\delta_{i}$ generally represents the importance of the element $i$ using its cardinality. In the majority processes are considered the formation of discussion or majority groups depending on similarities or distances among the expertsï $i \frac{1}{2}$ opinions. All values with a minimum of separation are considered inside the same group. The calculation method for the value $\delta_{i}$ is independent from the definition of the majority operators. The importance value $\delta_{i}$ can be calculated using the distance function:

$$
\operatorname{dist}\left(\left(a_{i}, \alpha_{i}\right),\left(a_{j}, \alpha_{j}\right)\right)=
$$

$$
\left\{\begin{array}{l}
1 \quad \text { if }\left|\Delta^{-1}\left(a_{i}, \alpha_{i}\right)-\Delta^{-1}\left(a_{j}, \alpha_{j}\right)\right| \leq x \\
0 \quad \text { otherwise }
\end{array}\right.
$$

Then, the cardinality of $\left(a_{i}, \alpha_{i}\right)$ is calculated as $\delta_{i}=\sum_{j=1, j \neq i}^{m} \operatorname{dist}\left(\left(a_{i}, \alpha_{i}\right),\left(a_{j}, \alpha_{j}\right)\right)$. The value $x$ models the final size of each group. 


\subsection{Results}

To test our proposal, an experimental study have been realized at the Faculty of Psychology in the University of Granada. In this study 26 tobacco addict people have rated a set of 11 slides from the IAPS on four dimensions: arousal, valence, dominance and desire. For each dimension a SAM scale with 9 human-like figures have been used. First, each human-like figure has been translated to an ordinal value from a linguistic variable $\mathcal{P}$ with 9 labels.

If we focus on the the ratings given by the participants on the slide with code 3266 on the dominance dimension we can observe the following values: $\{2,2,3,5,1,1,1,2,9,7,2,2,1,1,1,1,1,1,8,1$, $1,1,1,7,5,1\}$ where $73 \%$ of the values are lower or equal to 2 , and $54 \%$ are the value 1 . The classical aggregation operator (the mean) achieves an overall rate equal to 2.62 , whereas, the majority-based aggregation operator MA-LOWA $2 t$ produces a value equal to $\Delta^{-1}(2,-.34)=1.66$ which is a overall rate more related with the majority opinion. This value is computed as follows:

First, values $\{2,2,3,5,1,1,1,2,9,7,2,2,1,1,1$, $1,1,1,8,1,1,1,1,7,5,1\}$ are represented as 2-tuple values applying the $\Delta(\cdot)$ function, then, we obtain $\{(2, .0),(2, .0),(3, .0),(5, .0),(1, .0),(1, .0),(1, .0)$ $,(2, .0),(9, .0),(7, .0),(2, .0),(2, .0),(1, .0),(1, .0)$, $(1, .0),(1, .0),(1, .0),(1, .0),(8, .0),(1, .0),(1, .0)$, $(1, .0),(1, .0),(7, .0),(5, .0),(1, .0)\}$.

So, $\phi_{2 t}^{M A}((2, .0),(2, .0), \ldots,(5, .0),(1, .0))=$

$=W \cdot B^{T}=C_{2 t}^{7}\left\{w_{k}, b_{k}, k=1, \ldots, 7\right\}=$

$=w_{1} \otimes b_{1} \oplus\left(1-w_{1}\right) \otimes C_{2 t}^{6}\left\{\beta_{h}, b_{h}, h=2, \ldots, 7\right\}$.

where $B=[(3, .0),(8, .0),(9, .0),(5, .0),(7, .0),(2, .0)$, $(1, .0)]$ with cardinalities $[1,1,1,2,2,5,14]^{1}$, and $W=[0.005,0.005,0.005,0.038,0.038,0.205$, 0.705]. And for example, $w_{6}$ is computed as:

$$
\begin{aligned}
& w_{6}=\frac{\gamma_{6}^{\delta_{\min }}}{\theta_{\delta_{\max }} \cdot \theta_{\delta_{\min +2}} \cdot \theta_{\delta_{\min +1}} \cdot \theta_{\delta_{\min }}} \\
& +\frac{\gamma_{6}^{\delta_{\min +1}}}{\theta_{\delta_{\max }} \cdot \theta_{\delta_{\min +2}} \cdot \theta_{\delta_{\min +1}}}+\frac{\gamma_{6}^{\delta_{\min +2}}}{\theta_{\delta_{\max }} \cdot \theta_{\delta_{\min +2}}}+\frac{\gamma_{6}^{\delta_{\max }}}{\theta_{\delta_{\max }}} \\
& \text { with } \gamma_{6}^{\delta_{\min }}=1, \gamma_{6}^{\delta_{\min +1}}=1, \gamma_{6}^{\delta_{\min +2}}=1, \gamma_{6}^{\delta_{\max }}= \\
& 0, \theta_{\delta_{\max }}=2, \theta_{\delta_{\min +2}}=3, \theta_{\delta_{\min +1}}=5 \text {, and } \theta_{\delta_{\min }}= \\
& 7 \text {. } \\
& \text { So, } w_{6}=\frac{1}{2 \cdot 3 \cdot 5 \cdot 7}+\frac{1}{2 \cdot 3 \cdot 5}+\frac{1}{2 \cdot 3}=0.205 \text {. }
\end{aligned}
$$$$
14 .
$$

\section{Conclusions}

In this work, a new majority-based aggregation operator has been proposed and applying in a real psychological study. Ratings given by participants have been translated from the SAM scales to 2-tuple values and aggregated using the new majority-based aggregation operator, $M A-$ $L O W A_{2 t}$. Results show that overall rate produced by $M A-L O W A_{2 t}$ is a more consistence value with the majority concept than that produced by the mean (the classical aggregation operator used in these type of studies), avoiding the distribution problems.

Acknowledgments This work has been supported by the project Proyecto de Excelencia de la Junta de Andalucia SAINFOWEB, Cod. 00602.

\section{References}

[1] F. Aguilar de Arcos, M. Pérez, and M.B. Sánchez, Evaluación emocional en drogodependientes, Tech. report, Junta de Andalucía. Consejería de Asuntos Sociales, Comisionado para las Drogodependencias, 2003.

[2] M.M. Bradley and P.J. Lang, Affective reactions to acoustic stimuli, Psychophysiology 37 (2000), no. 2, 204-215.

[3] S. F. Coffey, M. Saladin, D.J. Drobes, K.T. Brady, B.S. Dansky, and D. G. Kilpatrick, Trauma and substance cue reactivity in individuals with comorbid posttraumatic stress disorder and cocaine or alcohol dependence, Drug and Alcohol Dependence 65 (2002), no. 2, 115127.

[4] P.C. Fishburn, A comparative analysis of group decision methods, Behavioral Science $\mathbf{1 6}$ (1971), 538-544.

[5] Center for the Study of Emotion and Attention [CSEA-NIMH], The international affective picture system: Photographic slides, Florida: The Center for Research in Psychophysiology, University of Florida, 1994.

[6] F. Herrera and E. Herrera-Viedma, Linguistic dcision analisys: Steps for solving decision problems under linguistic information, Fuzzy Sets and Systems 115 (2000), 67-82.

[7] F. Herrera, E. Herrera-Viedma, and J.L. Verdegay, Direct approach processes in group decision making using linguistic OWA operators, Fuzzy Sets and Systems 79 (1996), 175190.

[8] F. Herrera and L. Martínez, A 2-tuple fuzzy linguistic representation model for computing 
with words, IEEE Transactions on Fuzzy Systems 8 (2000), no. 6, 746-752.

[9] E. Herrera-Viedma, Modelling the retrieval process for an information retrieval system using an ordinal fuzzy linguistic approach, Journal of the American Society for Information Science and Technology 52 (2001), no. 6, 460475.

[10] E. Herrera-Viedma and A.G. López-Herrera, A fuzzy linguistic irs model based on a 2-tuple fuzzy linguistic approach, International Journal of Uncertainty, Fuzziness and KnowledgeBased Systems 15 (2007), no. 2, 225-250.

[11] P.J. Lang, The cognitive psychophysiology of emotion: Anxiety and the anxiety disorders, Hillsdale, NJ: Lawrence Erlbaum, 1985.

[12] _ The emotion probe: Studies of motivation and attention, American Psychology 50 (1995), 372-385.

[13] P.J. Lang, M.M. Bradley, and B.N. Cuthbert, Emotion, attention and the startle reflex, Psychological Review 97 (1990), 377-395.

[14] G. Pasi and R. Yager, Modeling majority opinion in multi-agent decision making, International Conference on Information Processing and Management of Uncertainty in Knowledge-Based Systems (IPMU02), France, 2002, pp. 1251-1257.

[15] M.C. Pastor, J. Moltó, J. Vila, and Lang. P.J., Startle reflex modulation, affective ratings and autonomic reactivity in incarcerated spanish psychopaths, Psychophysiology 40 (2003), no. 6,934 .

[16] J.I. Peláez and J.M. Doña, Majority additiveordered weighting averaging: A new neat ordered weighting averaging operators based on the majority process, International Journal of Intelligent Systems 18 (2003), 469-481.

[17] J. Vila, M. Sánchez, I. Ramirez, M.C. Fernández., P. Cobos, S. Rodriguez, M.A. Muñoz, M.P. Tormo, M. Herrero, P. Segarra, M.C. Pastor, S. Montañés, R. Poy, and J. Moltó, El sistema internacional de imágenes afectivas (IAPS): Adaptación española. Segunda parte, Revista de Psicología General y Aplicada 54 (2001), no. 4, 35-667.

[18] L.A. Zadeh, The concept of a linguistic variable and its applications to approximate reasoning. Part I, Information Sciences 8 (1975) 199-249, Part II, Information Sciences 8 (1975) 301-357, Part III, Information Sciences 9 (1975) 43-80. 